\title{
The Spectrum of Particles with Short-Ranged Interactions in a Harmonic Trap
}

\author{
Simon Tölle ${ }^{1,2, a}$, Hans-Werner Hammer ${ }^{1,2}$, and Bernard Ch. Metsch ${ }^{1,2}$ \\ 1 Helmholtz-Institut für Strahlen- und Kernphysik (Theorie), Nussallee 14-16, D-53115 Bonn Germany \\ 2 Bethe Center for Theoretical Physics, Universität Bonn, Nussallee 12, D-53115 Bonn Germany
}

\begin{abstract}
The possibility to control short-ranged interactions of cold gases in optical traps by Feshbachresonances makes these systems ideal candidates to study universal scaling properties and Efimov physics. The spectrum of particles in a trap, idealised by a harmonic oscillator potential, in the zero range limit with 2- and 3 -particle contact interactions is studied numerically. The Hamiltonian is regularised by restricting the oscillator basis and the coupling constants are tuned such that the ground state energies of the 2- and 3-particle sector are reproduced [1],[2]. Results for 2-, 3-, and 4 particle systems are presented and compared to exact results [3],[4].
\end{abstract}

\section{Introduction}

Cold atomic gases loaded in optical traps offer the possibility to investigate systems with strong interactions in a controlled manner. In the vicinity of Feshbach resonances, the scattering length can be tuned by an external magnetic field through a wide range [5]. For sufficiently large scattering length long-ranged properties, in particular the energy spectrum, are independent of the precise structure of the short-ranged potential. Sufficient means that the scattering length is the dominant part of the effective range expansion and the expansion can be truncated after the first term [6]. This situation coincides with the zero-range limit.

The three-body sector in free space is studied in [6]. In a non-relativistic effective field theory framework, one finds for three identical bosons an infinite sequence of 3atom bound states called Efimov states. These are geometrically distributed in the unitary limit.

The trap is idealised by a harmonic oscillator potential. In the zero range limit, the two-body sector is solved exactly by Busch [3]. The three-body sector is studied by Werner and Castin in the unitary limit [4]. We present a numerical procedure to study the three body sector also in the vicinity of the unitary limit. Furthermore, a possible extension to four particles is shown.

\section{Numerical Procedure}

For $A$ particles with equal masses the energy spectrum is determined by the Hamiltonian

$$
H=\sum_{i=1}^{A}\left(\frac{\left|\mathbf{p}_{i}\right|^{2}}{2 m}+\frac{1}{2} m \omega^{2}\left|\mathbf{x}_{i}\right|^{2}\right)+\sum_{i<j}^{A} V_{i j}+\sum_{i<j<k}^{A} W_{i j k}
$$

\footnotetext{
a e-mail: toelle@hiskp.uni-bonn.de
}

where $\omega$ is the trapping frequency and $V_{i j}$ and $W_{i j k}$ are 2and 3-particle contact interactions between particles $i, j$ and $k$.

In relative-(Jacobi)-coordinates

$$
\begin{gathered}
\mathbf{s}_{n}=\frac{1}{\sqrt{(n+1) n} \beta}\left(\mathbf{x}_{1}+\mathbf{x}_{2}+\ldots+\mathbf{x}_{n}-n \cdot \mathbf{x}_{n+1}\right), \\
n=1, \ldots, A-1, \\
\mathbf{R}=\frac{1}{\sqrt{A} \beta}\left(\mathbf{x}_{1}+\mathbf{x}_{2}+\ldots+\mathbf{x}_{A}\right),
\end{gathered}
$$

where $\beta=\sqrt{\frac{\hbar}{m \omega}}$ is the oscillator length, the Hamiltonian for the two body sector reads

$$
H_{\mathrm{cont}}^{(2)}=\frac{\hbar \omega}{2}\left(-\Delta_{\mathbf{s}_{1}}+\left|\mathbf{s}_{1}\right|^{2}-\Delta_{\mathbf{R}}+|\mathbf{R}|^{2}\right)+v \delta^{3}\left(\mathbf{s}_{1}\right) .
$$

\subsection{Two-Body Sector}

Since the interaction is independent of the centre of mass $\mathbf{R}$, the dynamics of the centre of mass separates. In order to solve the Schrödinger equation numerically, the Hilbert space is restricted by a cutoff parameter $N$ [1],[2]. The considered subspace is the linear span of oscillator functions $\phi_{n l m}\left(\mathbf{s}_{1}\right)$ with

$$
2 n+l \leq N .
$$

In this finite-dimensional subspace each matrix element

$$
\begin{aligned}
& \left\langle n l m\left|H_{\mathrm{cont}}^{(2)}\right| n^{\prime} l^{\prime} m^{\prime}\right\rangle= \\
& \int \mathrm{d}^{3} s \mathrm{~d}^{3} t\langle n l m \mid \mathbf{s}\rangle\left\langle\mathbf{s}\left|H_{\mathrm{cont}}^{(2)}\right| \mathbf{t}\right\rangle\left\langle\mathbf{t} \mid n^{\prime} l^{\prime} m^{\prime}\right\rangle
\end{aligned}
$$


is calculated. Since $\phi_{n m l}(0)=\langle n m l \mid 0\rangle=0$ for $l \neq 0$, the 2-particle contact interaction contributes for s-waves only,

$$
\begin{aligned}
& \left\langle n l m\left|H_{\text {cont }}^{(2)}\right| n^{\prime} l^{\prime} m^{\prime}\right\rangle= \\
& \hbar \omega(2 n+l) \delta_{n, n^{\prime}} \delta_{l, l^{\prime}} \delta_{m, m^{\prime}}+\frac{v}{\pi^{\frac{3}{2}}} f_{n} f_{n^{\prime}} \delta_{l, 0} \delta_{l^{\prime}, 0},
\end{aligned}
$$

with $f_{n}=\sqrt{\frac{(2 n+1) ! !}{n ! 2^{n}}}$. The time-independent Schrödinger equation thus becomes a finite-dimensional eigenvalue problem, which can be solved numerically.

Subsequently, the coupling constant is renormalised in the finite model space by the requirement that the ground state energy in the model space and in the full Hilbert space are identical. The separation of the interaction allows one to find the following relation between the ground state energy $E_{0}=\epsilon^{(2)} \hbar \omega$, the cutoff parameter $N$ and the coupling constant $v$ :

$$
-\frac{\pi^{\frac{3}{2}}}{v}=\sum_{n=0}^{\frac{N}{2}} \frac{\left|f_{n}\right|^{2}}{2 n+\frac{3}{2}-\epsilon^{(2)}} .
$$

For $\epsilon^{(2)}=-20$, Figure 1 shows the result for cutoff parameters $N \leq 800$. For the same $\epsilon^{(2)}$ and $N$ the eigenvalue

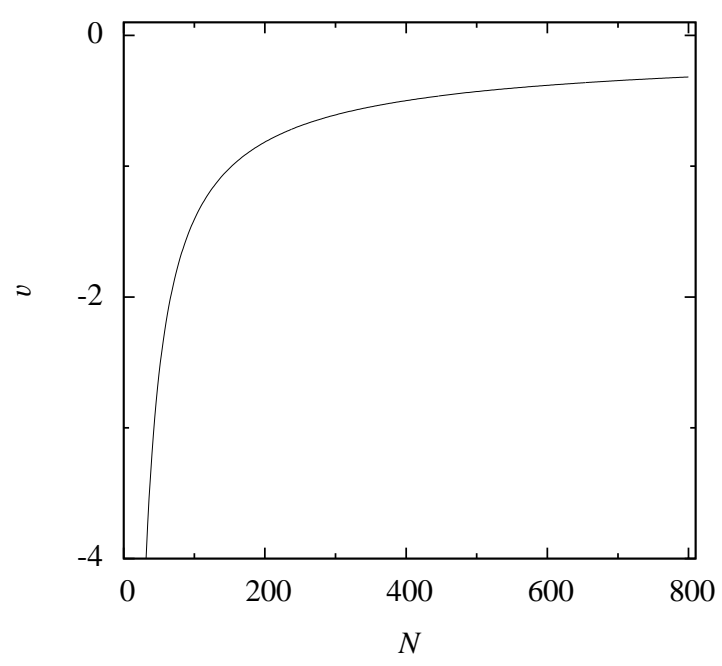

Fig. 1. Running Coupling $v$ depending on $N$

problem is solved. Figure 2 presents the energy spectrum for model spaces with $N \leq 800$ depending on $\kappa=\frac{1}{\sqrt{N+\frac{3}{2}}}$ for the angular momentum $l=0$. The exact spectrum derived by Busch [3] is added at the position $\kappa=0$.

Finally, the exact spectrum is numerically reproduced by extrapolating the results in the model spaces. We choose for the extrapolation function the ansatz

$$
f(\kappa)=a+b \kappa^{2}+c \kappa^{4}+d \kappa^{6} .
$$

Figure 2 shows that the exact spectrum can indeed numerically be determined in this manner.

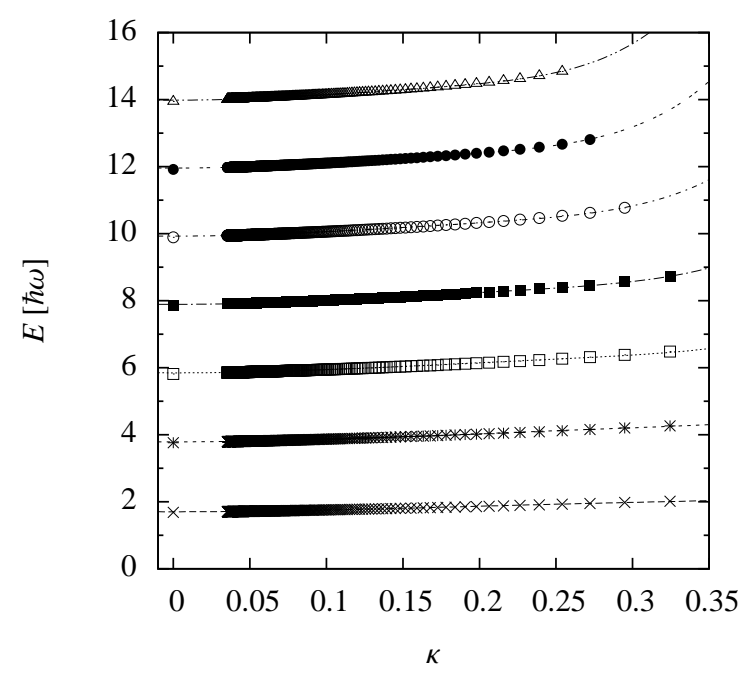

Fig. 2. Energy spectra for various size of the model space and extrapolation to $N \rightarrow \infty$

\subsection{Three-Body Sector}

The Hamiltonian in the three body sector $H_{\text {cont }}^{(3)}$ consists of three 2-particle contact interactions $V_{i}(i=1,2,3)$ and one 3-particle contact interaction $V_{\text {cont }}^{(3)}$. The procedure for solving the Schrödinger equation is analogous to the one used in the 2-body sector.

Firstly, a finite model space is constructed with a cutoff parameter $N$. The model space is the linear hull of the tensor products $\phi_{n_{1} l_{1} m_{1}}\left(\mathbf{s}_{1}\right) \otimes \phi_{n_{2} l_{2} m_{2}}\left(\mathbf{s}_{2}\right)$ with the restriction $2\left(n_{1}+n_{2}\right)+l_{1}+l_{2} \leq N$.

Secondly, all elements of the Hamilton matrix are calculated. In the three sets of Jacobi-coordinates,

$$
\begin{array}{ll}
\mathbf{s}_{1}^{(1)}=\frac{1}{\sqrt{2} \beta}\left(\mathbf{x}_{2}-\mathbf{x}_{3}\right), & \mathbf{s}_{2}^{(1)}=\frac{1}{\sqrt{6} \beta}\left(\mathbf{x}_{2}+\mathbf{x}_{3}-2 \mathbf{x}_{1}\right), \\
\mathbf{s}_{1}^{(2)}=\frac{1}{\sqrt{2} \beta}\left(\mathbf{x}_{1}-\mathbf{x}_{3}\right), & \mathbf{s}_{2}^{(2)}=\frac{1}{\sqrt{6} \beta}\left(\mathbf{x}_{1}+\mathbf{x}_{3}-2 \mathbf{x}_{2}\right), \\
\mathbf{s}_{1}^{(3)}=\frac{1}{\sqrt{2} \beta}\left(\mathbf{x}_{1}-\mathbf{x}_{2}\right), & \mathbf{s}_{2}^{(3)}=\frac{1}{\sqrt{6} \beta}\left(\mathbf{x}_{1}+\mathbf{x}_{2}-2 \mathbf{x}_{3}\right),
\end{array}
$$

the contributions of the potentials can readily be computed.

Except for $l_{\mathbf{s}_{1}}^{(i)}=0, l_{\mathbf{s}_{1}}^{(i)}=0, n_{\mathbf{s}_{2}}^{(i)}=n_{\mathbf{s}_{2}}^{(i)}$ and $l_{\mathbf{s}_{2}}^{(i)}=l_{\mathbf{s}_{2}}^{(i)}$, all contributions of $V_{i}$ vanish and thus

$$
\left\langle n_{\mathbf{s}_{1}}^{(i)} l_{\mathbf{s}_{1}}^{(i)}, n_{\mathbf{s}_{2}}^{(i)} l_{\mathbf{s}_{2}}^{(i)}\left|V_{i}\right| n_{\mathbf{s}_{1}}^{\prime(i)} l_{\mathbf{s}_{1}}^{(i)}, n_{\mathbf{s}_{2}}^{\prime(i)} l_{\mathbf{s}_{2}}^{\prime(i)}\right\rangle=\frac{v_{i}}{\pi^{\frac{3}{2}}} f_{n_{\mathbf{s}_{1}}^{(i)}} f_{n_{\mathbf{s}_{1}}^{(i)}} .
$$

Furthermore, the non-vanishing matrix elements of $V_{\text {cont }}^{(3)}$ are

$$
\left\langle n_{\mathbf{s}_{1}}^{(i)} l_{\mathbf{s}_{1}}^{(i)}, n_{\mathbf{s}_{2}}^{(i)} l_{\mathbf{s}_{2}}^{(i)}\left|V_{\text {cont }}\right| n_{\mathbf{s}_{1}}^{(i)} l_{\mathbf{s}_{1}}^{\prime(i)}, n_{\mathbf{s}_{2}}^{\prime(i)} l_{\mathbf{s}_{2}}^{(i)}\right\rangle=\frac{v^{(3)}}{\pi^{3}} f_{n_{\mathbf{s}_{1}}^{(i)}} f_{n_{\mathbf{s}_{1}}} f_{n_{\mathbf{s}_{2}}} f_{n_{\mathbf{s}_{2}}^{(i)}},
$$

with $l_{\mathbf{s}_{1}}^{(i)}=0, l_{\mathbf{s}_{1}}^{(i)}=0, l_{\mathbf{s}_{2}}^{(i)}=0$ and $l_{\mathbf{s}_{2}}^{(i)}=0$.

The remaining problem is to calculate the Hamilton matrix in one set of coordinates. 


\subsubsection{Talmi-Moshinsky-Transformation (TMT)}

The TMT provides a solution to this problem [7]. Under the Talmi-Transformation from the set of coordinates $(\rho$, $\lambda)$ to the set $\left(\rho^{\prime}, \lambda^{\prime}\right)$

$$
\left(\begin{array}{l}
\rho^{\prime} \\
\lambda^{\prime}
\end{array}\right)=\underbrace{\left(\begin{array}{ll}
\sqrt{\frac{1}{1+d}} & \sqrt{\frac{d}{1+d}}(-1) \\
\sqrt{\frac{d}{1+d}} & \sqrt{\frac{1}{1+d}}
\end{array}\right)}_{M} \cdot\left(\begin{array}{l}
\rho \\
\lambda
\end{array}\right), d \in \mathbb{R}, d \geq 0 .
$$

the transformation of the coupled oscillator functions depending on the first set to the ones depending on the second set is finite:

$$
\begin{gathered}
{\left[\phi_{n_{\rho} l_{\rho}}(\rho) \otimes \phi_{n_{\lambda} l_{\lambda}}(\lambda)\right]_{M_{L}}^{L}=\sum_{n_{\rho^{\prime}}^{\prime} l_{\rho^{\prime}}^{\prime} n_{\lambda^{\prime}}^{\prime} l_{\lambda^{\prime}}^{\prime}}} \\
\underbrace{\left\langle n_{\rho^{\prime}}^{\prime} l_{\rho^{\prime}}^{\prime}, n_{\lambda^{\prime}}^{\prime} l_{\lambda^{\prime}}^{\prime} ; L \mid n_{\rho} l_{\rho}, n_{\lambda} l_{\lambda}\right\rangle_{d}}_{\text {Brody-Moshinsky-Brackets }}\left[\phi_{n_{\rho^{\prime}}^{\prime} l_{\rho^{\prime}}^{\prime}}^{\beta}\left(\rho^{\prime}\right) \otimes \phi_{n_{\lambda^{\prime}} l_{\lambda^{\prime}}^{\prime}}^{\beta}\left(\lambda^{\prime}\right)\right]_{M_{L}}^{L} .
\end{gathered}
$$

Under this transformation the total energy, the total angular momentum and the parity are conserved. Sets of Jacobi coordinates can be transformed by Talmi transformations into each other and the Hamilton matrix can be calculated in one set of Jacobi coordinates.

The three coupling constants $v_{i}^{(2)}, i=1,2,3$, of the 2 particle interactions are renormalised by matching to three given ground state energies of the two body sector with relation (8). In order to determine the renormalisation of the 3-body coupling $v^{(3)}$, we use the separability of $V_{\text {cont }}^{(3)}$. In the model space, the eigenvalue problem without this 3 -particle interaction is solved. With the eigenvector

$$
|k\rangle=\sum_{n_{\mathbf{s}_{1}}^{\prime} l_{\mathbf{s}_{1}}^{\prime}, n_{\mathbf{s}_{2}}^{\prime} l_{\mathbf{s}_{2}}^{\prime}, L}^{N} Z\left(k ; n_{\mathbf{s}_{1}}^{\prime} l_{\mathbf{s}_{1}}^{\prime}, n_{\mathbf{s}_{2}}^{\prime} l_{\mathbf{s}_{2}}^{\prime}, L\right)\left|n_{\mathbf{s}_{1}}^{\prime} l_{\mathbf{s}_{1}}^{\prime}, n_{\mathbf{s}_{2}}^{\prime} l_{\mathbf{s}_{2}}^{\prime}, L\right\rangle .
$$

corresponding to the energy eigenvalue $D(k)$, the following dependence between $N$ and $v^{(3)}$ results:

$$
-\frac{\hbar \omega \pi^{3}}{v^{(3)}}=\sum_{k} \frac{\left|\sum_{n_{\mathbf{s}_{1}}, n_{\mathbf{s}_{2}}} Z\left(k ; n_{\mathbf{s}_{1}}, n_{\mathbf{s}_{2}}\right) f_{n_{\mathbf{s}_{1}}} f_{n_{\mathbf{s}_{2}}}\right|^{2}}{D(k)-\epsilon^{(3)}} .
$$

In the model space with a given cutoff parameter $N$, the finite eigenvalue problem corresponding to the full Hamiltonian can then be solved.

\subsubsection{Unitary limit}

For the present we are interested in the unitary limit. The corresponding ground state energy for the 2-particle sector is $\epsilon^{(2)}=\frac{1}{2}$ [3]. Because the three coupling constants $v_{i}^{(2)}$ are identical, the Hamiltonian is invariant under permutation of particles. It follows that the Hamilton matrix is block diagonal in different symmetries of wave functions.

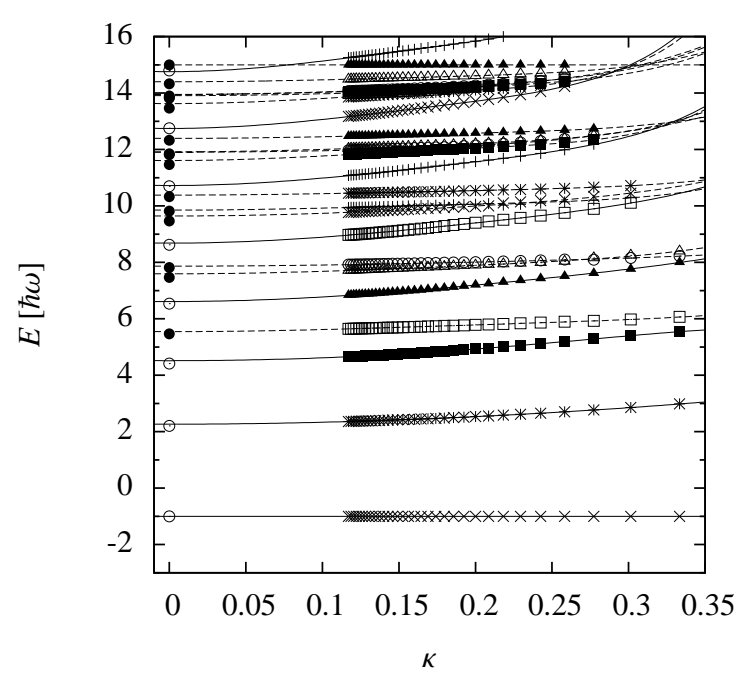

Fig. 3. For $\epsilon^{(3)}=-1$ spectrum for model spaces with $N \leq 70$, polynomial extrapolations and exact results for Efimov (open circles) and non Efimov states (dots) in the unitary limit at $\kappa=0$.

For completely symmetric wave functions, i.e. 3 identical bosons, Figure 3 shows (for $N \leq 70$ ) the spectrum depending on $\kappa=\frac{1}{\sqrt{N+3}}$ for a chosen state energy $\epsilon^{(3)}=-1$, positive parity and total angular momentum $L=0$. There are two different types of states. On the one hand, there are states independent of $V^{(3)}$ (dashed). On the other hand, there are states which depend on $V^{(3)}$ (solid) and are called Efimov states. Additionally, the exact results determined by Werner and Castin [4] are given at $\kappa=0$ and the spectrum is extrapolated to $N \rightarrow \infty$ with the same ansatz as in the two body sector. A comparison to the exact results proves the extrapolation to be reliable.

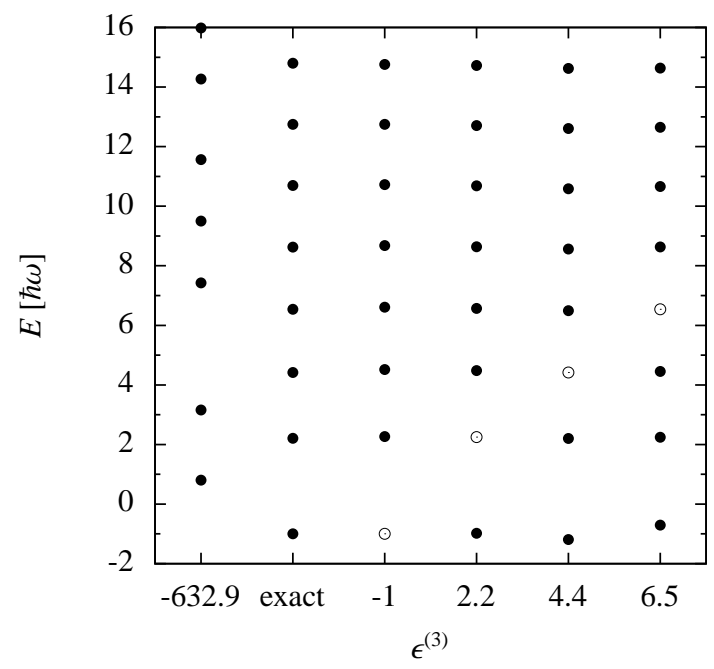

Fig. 4. Exact spectrum and extrapolated spectra of Efimov states for various $\epsilon^{(3)}$. The renormalised energies $\epsilon^{(3)}$ are marked by open circles. 
The exact spectrum of Efimov states is fixed by energy of one 3-body state. It does not matter which state in a spectrum is chosen. The extrapolated spectra should have this property, too. In Figure 4 the exact spectrum and the extrapolated spectra of Efimov states for various energies $\epsilon^{(3)}$ are shown. For small $\epsilon^{(3)}$, the spectra coincide. For $\epsilon^{(3)} \approx-632$, which is the next state below $\epsilon^{(3)}=-1$, the extrapolated spectrum deviates from the exact result.

\subsubsection{Finite Scattering Lengths}

Beginning with the relation between the ground state energy $\epsilon^{(2)}$ and the scattering length $a_{0}$ in the 2 particle sector found by Busch [3], the spectra for 3 identical bosons with finite scattering length can be determined. Figure 5 shows this relation and the scattering lengths considered are flagged by arrows.

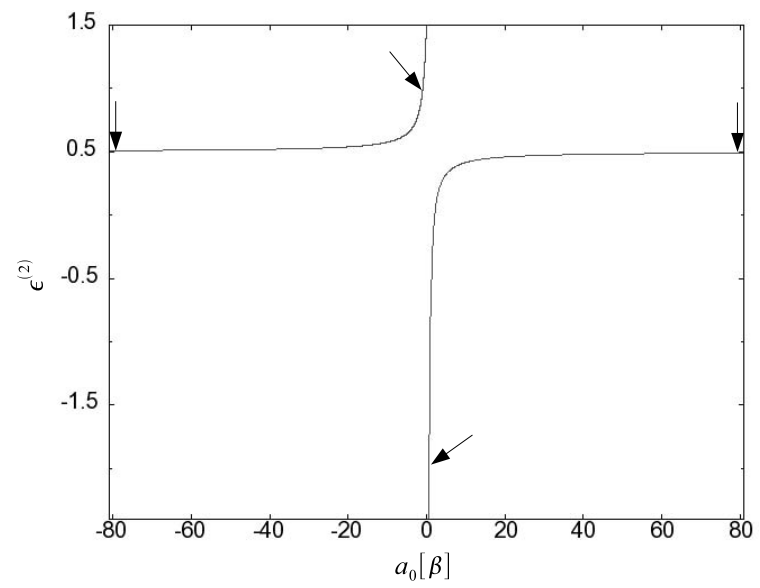

Fig. 5. Relation between $\epsilon^{(2)}$ and $a_{0}$ in units of the oscillator length $\beta$. Arrows highlight the considered scattering lengths.

According to the procedure described above the energy spectrum is determined for this four scattering lengths by restricting the Hilbert space, renormalising the coupling constants, solving the eigenvalue problem and extrapolating to $N \rightarrow \infty$.

$\epsilon^{(3)}$ is fixed at -1 . As shown in Figure 6, the results for the large scattering lengths $\pm 80 \beta$ coincide almost with those in the unitary limit. For the small positive scattering length, the spectrum is shifted downwards and for the small negative scattering length, it is shifted in the opposite direction. In the future, the dependence should be studied in more detail.

\subsection{Four-body sector}

The numerical method is extendable to four particles. Consider two identical fermions with two other particles. The wave function is antisymmetric under permutation of identical fermions. Therefore, there is no four particle contact interaction and no contact interaction between two

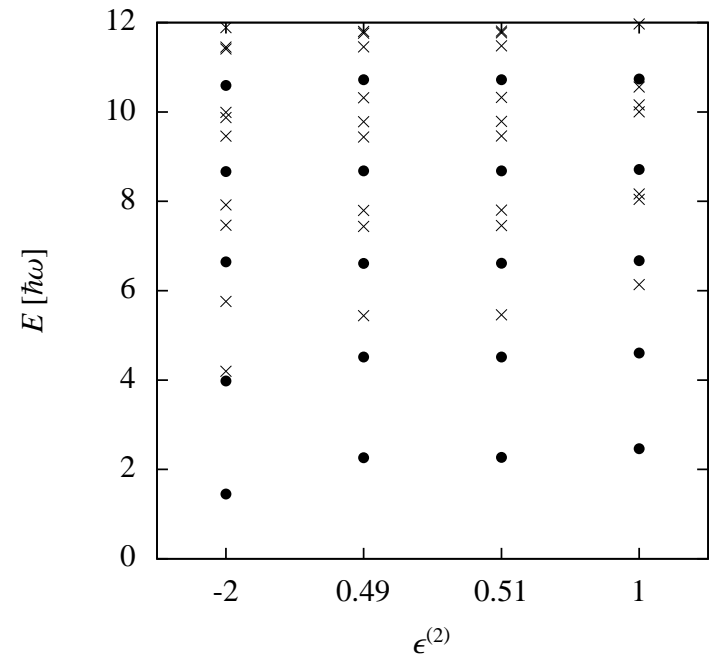

Fig. 6. Extrapolated spectra for Efimov (dots) and Non-Efimov states (crosses) for various $\epsilon^{(2)}$

fermions exists. Altogether, there are five 2-particle contact interactions and two 3-particle contact interactions. Keep in mind that the contributions of some interactions are pairwise identical because two of the particles are identical.

The model space is the linear span of the tensor product of one particle wave functions depending on Jacobicoordinates, $\mathbf{s}_{1}, \mathbf{s}_{2}, \mathbf{s}_{3}$, for four particles with $N \geq 2\left(n_{1}+\right.$ $\left.n_{2}+n_{3}\right)+l_{1}+l_{2}+l_{3}$. Again, the Hamilton matrix is searched on this model space. In order to determine all contributions of the interactions in one set of Jacobi-coordinates, the TMT is used again. The transformation is defined for coupled wave functions. Since the coupling is not associative, one additionally needs $6 \mathrm{j}$-symbols for the recoupling of the wavefunction [8]. Analogous to the three-body sector, the four coupling constants of the 2-particle interactions are renormalised by equation (8) and the two coupling constants of the 3-particle interactions by equation (15) for given ground state energies $\epsilon^{(2)}$ and $\epsilon^{(3)}$. The finite eigenvalue problem is solved and an approximation for the energy spectrum in the full Hilbert space is determined by the extrapolation to $\kappa=\frac{1}{\sqrt{N+\frac{9}{2}}} \rightarrow 0$ with an appropriate ansatz. Due to the time required for computing the eigenvalues for great cutoff parameter, $N$ must be chosen less or equal 20. Therefore we take the ansatz $f(\kappa)=a+b \kappa^{2}+c \kappa^{4}$ instead of the symmetric polynomial of order six as in the three body sector.

In Figure 7, the extrapolated spectrum is shown for various ground state energies $\epsilon^{(3)}$ in the unitary limit. The total angular momentum $L$ is zero and the parity is positive. There are two different types of states: states dependent on $\epsilon^{(3)}$ (dots) and states independent of $\epsilon^{(3)}$ (crosses). The former depend linearly on $\epsilon^{(3)}$

\section{Outlook}

In the future the three particle sector with finite scattering lengths should be studied in more detail. In particular, the 


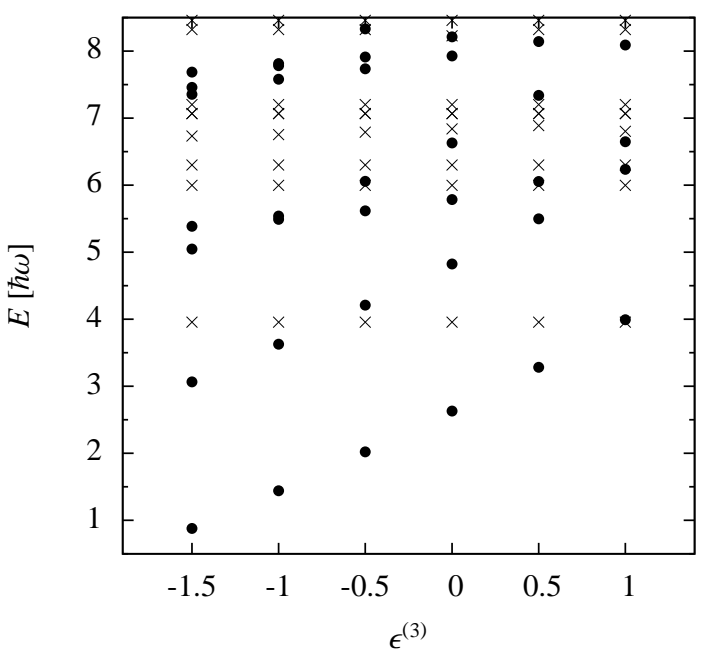

Fig. 7. Extrapolated spectra for Efimov (dots) and Non-Efimov states (crosses) for various $\epsilon^{(2)}$

dependence of the energy spectrum on the scattering length should be investigated. Furthermore, we want to look at systems with mixed symmetries. Its possible to make predictions for physical systems, e.g., a gas of three ${ }^{6} \mathrm{Li}$ atoms loaded in an optical lattice. These atoms are fermion-like and if they are in two different hyperfine states, the wave function is mixed antisymmetric.

In principal, the numerical procedure can be extended to compute the spectrum for four and more identical bosons. The difficulty lies in the construction of the wave functions in the different sets of Jacobi-coordinates with the correct symmetry. Here, the TMT and the recoupling are used again. Moreover, the number of states for a given cutoff parameter heavily increases with the number of particles. Thus, the computation of the spectrum becomes more expansive so that only spectra for small cutoff parameters can be determined.

Up to now, only the limit of a deep trapping potential at each lattice site is considered. In order to describe an experimental scenario with shallower potentials at each site, additional effects must be considered. These include tunneling of atoms between different sites and interactions between atoms at different sites.

\section{References}

1. Y. Alhassid et al., Phys. Rev. Lett. 100(23), 230401 (2008)

2. I. Stectu et al., Phys. Lett. B 653, 358 (2007)

3. T. Busch et al., Found. Phys. 28, 549 (1998)

4. F. Werner, Y. Castin, Phys. Rev. Lett. 97, 150401 (2006), cond-mat/0507399

5. E. Tiesinga et al., Phys. Rev. A 46, R1167 (1992)

6. E. Braaten, H.W. Hammer, Phys. Rep. 428, 259 (2006)

7. T.A. Brody, Tablas de Paréntesis de Transformación (Universidad Nacional Autonoma de Mexiko, 1960)
8. D.A. Varshalovich et al., Quantum Theory of Angular Momentum, 2nd edn. (World Scientific Publishing, 1988) 